\title{
Análisis de las ventajas y desventajas del sistema de gestión de la seguridad de la información y su influencia en la competitividad de las empresas que utilizan Cloud Computing y Big Data en el Ecuador
}

\section{Analysis of the advantages and disadvantages of the information security management system and its influence on the competitiveness of companies that use Cloud Computing and Big Data in Ecuador}

Ing. Ricardo Rafael Coello Yagual

Universidad Internacional del Ecuador, Ecuador

Lucía Magdalena Pico Versoza

Universidad Internacional del Ecuador, Ecuador

Autor para correspondencia: ricoelloya|@uide.edu.ec, lupicove@uide.edu.ec

Fecha de recepción: 30 de octubre de 2017 - Fecha de aceptación: 15 de Abril de 2018

\section{Resumen}

El presente trabajo de investigación refleja el análisis de las ventajas y desventajas de la seguridad de la información, en el proceso de toma de decisiones de las empresas que utilizan Cloud Computing y Big Data en el Ecuador. Para realizar el análisis, se identificó las variables esenciales que influyen en el proceso de toma de decisiones. Así también se presenta el estudio de la Norma ISO 217001 como un elemento importante en la propuesta de la implementación del Sistema de Seguridad de la Información en las organizaciones, como una herramienta de protección de los datos estructurados y no estructurados que proceden del Big Data y el Cloud Computing.

Palabras Claves: big data; cloud computing; sistema de seguridad de la información; norma ISO 27001

\begin{abstract}
This research work reflects the analysis of the advantages and disadvantages of information security in the decision, making process of companies that use Cloud Computing and Big Data in Ecuador. To carry out the analysis, the essential variables that influence the decision-making process were identified. The study of ISO 217001 is also presented as an important element in the proposal for the implementation of the Information Security System in organizations, as a tool to protect structured and unstructured data that come from Big Data and Cloud Computing.
\end{abstract}

Key Words: big data; cloud computing; information security system; ISO 27001 


\section{Introducción}

En la actualidad las empresas se encuentran en una búsqueda de ventajas competitivas que las lleve a perdurar en el tiempo, este fenómeno se fundamenta en buscar continuamente satisfacer las necesidades del cliente, es decir que el proceso de toma de decisiones tiene una gran importancia en la creación valor para la organización.

La empresa actualmente busca mayores posibilidades de éxito y mantenerse por delante de su competencia, es decir la influencia de la globalización ha generado consumidores cada día más exigentes, así también "depurar" los requerimientos que solicitan los clientes se convierte para la empresa en una prioridad de éxito en la consecución de los resultados que espera (Martinez \& Lara, 2014). Las organizaciones necesitan saber sobre el entorno que las rodea, por ello se vuelve muy importante la buena interpretación de la información que proviene de las diferentes fuentes que se manejan actualmente; ante ello las tecnologías de la información y la comunicación se han convertido en las herramientas que las empresas utilizan no solo para adquirir tecnología, sino que actualmente son estrategias competitivas que intervienen en el proceso de la toma de decisiones de las empresas. A través del proceso de investigación se encontró los siguientes requerimientos:

...estabilidad macroeconómica, apertura y acceso a mercados internacionales o la complejidad de la regulación para el sector empresarial, la infraestructura regional y la competitividad de las empresas (Bonales \& Zamora, 2015).

En el presente estudio a través de la investigación bibliográfica se hace referencia a una parte importante del tercer nivel, es decir a las herramientas que se pueden utilizar para protección de los datos tanto estructurados como no estructurados que provienen de fuentes masivas como el Big Data y el Cloud Computing; en este caso se analizará la propuesta del Sistema de Seguridad de la Información, como una alternativa para la protección y custodio de la información valiosa que la organización necesita.

Siguiendo nuestra investigación se podría indicar que el volumen de datos generados por diferentes fuentes de información crece exponencialmente, sólo en los últimos dos años la cifra mundial ha aumentado un 92\% (Tascán, 2013).

\section{La era de la información}

En la era de la información, de la explosión de sus tecnologías, se vive la etapa en la que la humanidad ha alcanzado un desarrollo imprevisible; cada día son mayores las diferencias sociales, políticas y económicas (Quiroga, 2002). Se puede indicar entonces sobre la sociedad de la información, que es visible el paso de las sociedades industriales a las posindustriales y del conocimiento, donde el factor esencial de progreso es el conocimiento. Esta nueva sociedad, con organizaciones basadas en el aprendizaje, cuyo su capital más preciado es el ser humano, se sustenta en un desarrollo tecnológico sin precedentes, es el punto en el cual las grandes compañías planifican sus productos en función de la gestión del conocimiento y de la viabilidad para su obtención. En este contexto, debe entenderse que las tecnologías de información y las telecomunicaciones no son más que un medio para transmitir y gestionar datos, información y conocimiento, el conocimiento es factor fundamental para la creación de riquezas (Quiroga, 2002). 
En la era de la información, uno de los principales problemas es su exceso, es necesario invertir mucho tiempo en ella debido, entre otras causas, a la liberación de los mecanismos regulatorios existentes en materia de publicaciones, sobre todo como resultado del surgimiento y desarrollo de Internet; por esta razón, es frecuente encontrar un número significativo de publicaciones redundantes y de baja calidad mezcladas con otras importantes y sólidas, difíciles de hallar entre la información ruidosa que la "envuelve". Son múltiples las definiciones de un mismo concepto, por ejemplo, existe una multitud de definiciones, que en su conjunto son bastante dispares y, en muchos casos, ambiguas

\section{La Norma ISO 27001 - aspectos generales}

La norma ISO 27001 ofrece los requisitos básicos para implementar un Sistema de Gestión de Seguridad de la Información.

A través del estudio de la norma se encontró que información es "el conjunto de datos organizados en poder de una organización que poseen valor para la misma, independientemente de la forma en la que se guarde o retrasmita su origen o fecha de elaboración” (Fernandez \& Piattini, 2012).

De la norma ISO 27001 se encontró que La seguridad de la información consiste en la preservación de la confidencialidad, integridad y disponibilidad, además de los sistemas que se encuentran implicados en su tratamiento, dentro de la empresa.

Es necesario mencionar que la norma ISO 27001 puede ser implementada en las organizaciones para mejorar la seguridad de la información (Fernandez \& Piattini, 2012). En el momento en el que se decide implementar la norma se debe seguir las siguientes recomendaciones:

- Mantener la sencillez y restringir el alcance. Un centro de trabajo, un proceso de negocio, un único centro de proceso de datos o un área sensible concreta, una vez que se ha conseguido el éxito y se han observado los beneficios, es necesario que se amplíe de forma gradual el alcance en diferentes fases.

- Comprender los detalles que tiene el proceso de implementación. Es necesario que se inicie en base a las cuestiones exclusivas técnicas, siendo un error sobrecargar de problemas la implementación. Es bueno adquirir experiencia de otras implementaciones, asistir a cursos de formación o contar con el asesoramiento de consultores externos especializados.

- Gestionar el proyecto fijando los diferentes hitos con los objetivos y los resultados.

- La autoridad y el compromiso que ha tomado la dirección de la organización evita las excusas para desarrollar las buenas prácticas, además de ser uno de los puntos fundamentales de la norma ISO 27001.

- La certificación como objetivo. Aunque se pueda conseguir la conformidad con la norma sin certificarse. La certificación asegura un mejor enfoque, un objetivo mucho más claro y tangible e incrementa las opciones de conseguir el éxito. La certificación no debe ser la única meta. El principal objetivo es la gestión de la seguridad de la información que se alinea con el negocio. 
- No inventarse nada. Es bueno apoyarse en los estándares, métodos y guías ya establecidos, además de la experiencia de otras empresas.

- Pueden ser otros sistemas de gestión también implementado como ISO 9001, ISO 14001, etc. Pueden resultar útil como estructura de trabajo, ahorra tiempo y esfuerzo, además de crear sinergias. Es conveniente pedir ayuda e implicar a los responsables y auditores internos de otros sistemas de gestión.

- Reservar la dedicación necesaria al día o a la semana. El personal involucrado en el proyecto debe ser capaz de trabajar con continuidad en el proyecto.

- Registrar las evidencias. Deben recogerse evidencias al menos tres meses antes de realizar la auditoría de certificación, para demostrar que el Sistema de Gestión de Seguridad de la Información funciona de forma correcta.

- Mantener y mejorar de forma continua. Es necesario considerar el mantenimiento y la mejora del Sistema de Gestión de Seguridad de la Información a lo largo del tiempo, esto supone que se requiere de esfuerzo y dedicación.

\section{Planteamiento y formulación del problema}

A través del planteamiento y formulación del problema se evidencia la existencia de ventajas y desventajas del Sistema de Seguridad de la Información en el proceso de la toma de decisiones de las empresas. Entre los síntomas más importantes que se encontraron tenemos:

- Los datos no estructurados son elementos de información y se han convertido ventaja competitiva para la organización, así también para las pymes se han constituido en el motor de desarrollo comercial (El Comercio, 2015), por ello es necesario la existencia de herramientas que ayuden en la protección de la información proveniente del Big Data y el Cloud Computing.

- La web, los medios sociales y los datos estructurados se han convertido junto con las herramientas de inteligencia de negocios en índices de competitividad en el Ecuador. (El Universo, 2016), por ello es necesario el análisis de las ventajas y desventajas que se presentarían en la implementación de un Sistema de Seguridad de la Informaciones en las organizaciones.

Siguiendo la línea de investigación se encontró las causas que afectan a los síntomas anteriormente expuestos, entre ellas tenemos:

- Actualmente la empresa emergente digital recibe la información que proviene de la web semántica y los medios convencionales de información (datos estructurados), pero dentro del mundo del mundo de los negocios, el conocer las necesidades de los clientes, responder a sus inquietudes se ha vuelto un indicador de competitividad y la venta de sus productos y servicios permite el crecimiento económico de las mismas. (Crespo, 2013), siendo una causa importante la implementación de un Sistema de Seguridad de la Información que sirva para protegerla.

- El Big Data y el Cloud Computing se convierten en una ventaja para los negocios que buscan comprender y analizar continuamente la interacción con sus clientes, es decir la creación de nuevas oportunidades de negocios (Aguirre \& Andrade, 2006), lo cual ha permitido el incremento en sus ingresos, lo cual se constituye en una información valiosa, indispensable para la propuesta que busca implementar este estudio. 
Utilizando el formato de investigación se presentan las siguientes preguntas de pronóstico:

- ¿Cuál debería ser el papel que realice el Sistema de Seguridad de la Información, como la herramienta encargada por la organización para proteger los datos estructurados y no estructurados que provienen de las fuentes de información como Big Data y Cloud Computing?

- ¿Qué ventajas y desventajas tendría en los recursos disponibles de las empresas en la implementación de Sistema de Seguridad de la Información?

Se encontró entonces, el siguiente control del pronóstico:

- Actualmente las empresas interpretan la información no estructurada que proviene de los medios sociales, video y audio a través del Big Data y el Cloud Computing, por ello es necesario registrar las evidencias. Deben recogerse evidencias al menos tres meses antes de realizar la auditoría de certificación, para demostrar que el Sistema de Gestión de Seguridad de la Información funciona de forma correcta.

- El comercio electrónico y la competitividad exigen a las empresas adoptar una postura global que busque incrementar sus ingresos. Por ello la implementación herramientas que custodien los datos masivos proveniente de estas fuentes de información, se debe priorizar la implementación de un Sistema que busque la protección de los datos.

El pronóstico y control del pronóstico permitieron realizar la formulación del problema de investigación, que se presenta a continuación:

\section{¿Cuáles son las ventajas y desventajas de la implementación del Sistema de Seguridad de la Información y su influencia en la competitividad de las empresas que utilizan Big Data y Cloud Computing?}

A través de la metodología escogida se estableció el siguiente objetivo general:

- Analizar las ventajas y desventajas de la implementación del Sistema de Seguridad de la Información en la competitividad de las empresas que utilizan Big Data y Cloud Computing.

Estableciendo los siguientes objetivos específicos:

- Determinar las ventajas y desventajas que obtienen las empresas con la implementación de Normas ISO 27001

- Proponer la implementación del Sistema de Seguridad de la Información para la protección de la información masiva que proviene del Big Data y el Cloud Computing.

\section{Justificación teórica de la investigación}

El trabajo de investigación surgió de la necesidad de establecer las ventajas y desventajas que se obtienen de la implementación del Sistema de Seguridad de la Información, el cual protegen los datos no estructurados provenientes del Big Data y el Cloud Computing, es necesario indicar que los datos masivos facilitan el conocimiento de las necesidades del cliente, creando valor y obteniendo competitividad indispensable para el desarrollo organizacional (Aguirre \& Andrade, 2006). 
Siguiendo la metodología seleccionada se presenta el esquema del método de las variables (gráfico 1) para establecer la influencia del social media en la competitividad de los negocios.

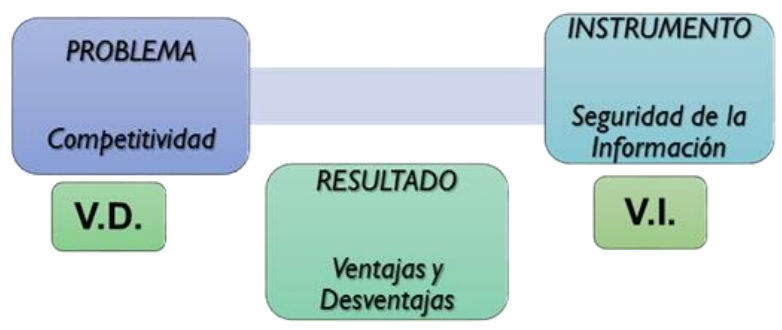

Gráfico 1. Método de las variables Fuente: Elaboración propia

\section{Desarrollo}

Big Data apareció en el año 2012 como uno de los términos de moda en todas las revistas de temática científica, social o tecnológica, apareció también en blogs y redes sociales e incluso ya ha dado el salto a las publicaciones económicas y empresariales y las de divulgación más popular (Tascán, 2013). El Big Data, aunque su nombre hace referencia a la cantidad de datos, el tamaño y el número no son las únicas variables gigantes que están implicadas.

\section{La era del Big Data}

La sociedad se encuentra en la era del Big data, que se manifiesta por la generación de grandes cantidades de datos estructurados y no estructurados en tiempo real o diferido, los cuales necesitan ser analizados por las empresas para crear valor, esta era se caracteriza por lo dinámico de la creación de la información, es decir que "no sólo por el tamaño del conjunto de datos que se encuentran disponibles, sino que también posee otros atributos” (Martinez \& Lara, 2014); así también los autores identifican tres elementos que caracterizan a este fenómeno, llamados como: el volumen, la velocidad y la variedad. Algunos autores añaden un cuarto valor que es conocido como la veracidad. Es importante comprender que además de los datos estructurados, que son aquellos que provienen de fuentes de información conocidas, considerados también fáciles de medir y analizar por medio de los sistemas tradicionales, existen otros en la actualidad conocidos como los datos no estructurados, que son aquellos que provienen de la web, de las cámaras de los móviles y videos, de las redes sociales, de los sensores de las ciudades y edificios.

Las empresas en la actualidad presentan una gran dificultad para analizar estos datos no estructurados ya sea por la rapidez con la que se generan o por el volumen de contenido que poseen (Tascán, 2013). En el universo empresarial y el mundo de los negocios, se ha utiliza mucho el término Business Intelligence (BI) que no es otra cosa que el conjunto de estrategias y herramientas que una empresa tiene a su disposición para poder analizar los datos de su organización (Cueva, 2014), actualmente con las herramientas BI se realizan predicciones y análisis.

Big Data se lo relaciona con la minería de datos, que "es un campo de las Ciencias de la Computación que intenta descubrir los patrones en grandes volúmenes de datos” (Tascán, 2013), la minería de datos que es parte del BI, utiliza elementos de inteligencia artificial y 
estadística para la depuración de la información, este aspecto lo relaciona al Big Data que utiliza también este procedimiento para crear valor de la información.

Así como las tecnologías mejoran exponencialmente, así también las herramientas habituales de trabajo cambian, es así como el talento humano de las organizaciones pasó de la "hoja de Excel a Hadoop, un software que permite trabajar miles de nodos distribuidos y con peta bytes de información" (Tascán, 2013)

El Cloud Computing - una solución para la gestión en los negocios

La tecnología evoluciona de manera acelerada haciendo que los programas que posee la empresa queden obsoletos rápidamente, en ocasiones la inversión que realizó la compañía no alcanzó a recuperarla, ya que es una de las primeras que debe realizar y, en muchas ocasiones una de las que más parte del presupuesto requiere (Abelar \& Hernández, 2010)

En la actualidad el modelo de negocio de Cloud Computing es una ventaja competitiva para la organización que busca "racionalizar el gasto en tecnología de la información permitiendo concentrar los recursos financieros y de personal en la actividad principal del negocio" (Abelar \& Hernández, 2010), siendo este un factor que permite la creación de valor y sostenimiento de la organización,

... al no tener como prioridad disponer de mucho dinero en el montaje de la empresa, proporcionándoles una infraestructura robusta y confiable para la gestión de manejo de datos, pues sólo se necesitan computadores de características normales y conexión a internet para el uso de los programas que necesiten (Mamani, 2014)

Cloud Computing es una estrategia de bajo costo y facilidad para la organización, que se ha convertido en una buena alternativa que apoya el emprendimiento al permitirle a los miembros de la empresa que "sólo se concentren en el objeto del negocio, sin que los sistemas de información se conviertan en un problema de tiempo, conocimiento o dinero" (Abelar \& Hernández, 2010)

Para implementar Cloud Computing en la empresa es importante conocer el negocio, es decir saber que existen procesos, servicios requeridos y ofrecidos, flujos de información y quienes intervienen en ellos, lo cual permitirá obtener la mejor decisión en la búsqueda de optimizar la gestión de la información, esto también nos brindará un análisis mayor en lo que se necesita en términos de infraestructura, plataforma y software (Mamani, 2014).

Es importante indicar que el Cloud Computing es una metodología de trabajo ya que por medio de la combinación de varias tecnologías de la información ya existentes y diferentes procedimientos, permite una nueva forma de hacer el trabajo dentro de una organización (Abelar \& Hernández, 2010)

Así tenemos que la Asociación Española para la calidad define a la seguridad de la información como: "La Seguridad de la Información tiene como fin la protección de la información y de los sistemas del acceso, uso, divulgación, interrupción o destrucción no autorizada” (Valarezo, 2013) 


\section{Big Data y su creación de valor en los negocios}

La transformación que se ha dado con la llegada de la Web 2.0 ha popularizado aspectos de la informática tales como Social Computing o "software social" y la nube informática o "Cloud Computing", actualmente el volumen de datos que se generan en nuestra Sociedad de la Información y el Conocimiento (SIC) son abundantes y con un crecimiento exponencial que se genera segundo a segundo. La denominación que predomina en la actualidad asociada a la problemática de gestionar estas grandes cantidades de datos que no se pueden tratar con medios tradicionales (Calero, 2013), es Big Data. El estudio de grandes cantidades de datos tomando como referencia estas propiedades permite la identificación rápida de patrones que ayudan a las empresas en el proceso de la toma de decisiones, a través de la minería de los datos, que se ha convertido en una herramienta muy valiosa de crecimiento empresarial en el mundo de los negocios.

Es necesario saber que los datos por sí mismos aportan nada o incluso generar ruido, especialmente si se encuentran en volúmenes elevados, por lo que su procesado se hace indispensable (Martinez \& Lara, 2014). Por ello la importancia de esta herramienta ya que permite a la empresa crear valor de los datos no estructurados, esto genera una información valiosa y necesaria para el administrador de empresas. Con la llegada de la web 2.0 y el protagonismo que adquiere el usuario, es decir han aumentado (Martinez \& Lara, 2014).

Algunas cifras permiten medir la magnitud de tal crecimiento. Según ComScore, "desde el 2007 al 2011 la audiencia mundial en sitios sociales registra un aumento del 174\% y en junio del 2012 sumaban 1.258 millones de usuarios" (Martinez \& Lara, 2014). Los datos por plataformas también resultan espectaculares por su dimensión, tenemos por ejemplo que en marzo del 2014 Facebook contaba con 802 millones de usuarios activos al día y, la comunidad de profesionales que se conectan a través de LinkedIn sumaba en abril de ese año, más de 300 millones de miembros (Tascán, 2013).

Atendiendo al intercambio de contenidos destacan los 500 millones de tweets al día que registra Twitter, las 100 horas de vídeo que cada minuto se suben a YouTube y los 25 mil millones de 'pins' que se contabilizan en Pinterest (Martinez \& Lara, 2014)

La información y los mensajes que se intercambian en los Social Media, videos y audios que circulan en una dimensión semipública de la comunicación tienen perfiles de privacidad, por lo cual esta información no puede ser utilizada en su totalidad por las empresas, esto ha generado un debate sobre el respecto del derecho a la intimidad. Siguiendo nuestra línea de investigación abordaremos las aplicaciones que se obtiene de la implementación del Cloud Computing.

\section{Capas del Cloud Computing}

El Cloud Computing se puede dividir en tres niveles (capas) en función de los servicios que actualmente están ofreciendo las empresas, en el futuro pueden surgir otras capas o clasificaciones. 
- Infraestructura como servicio: Se puede decir que es la parte física de la nube. En lugar de que los clientes tengan sus equipos en su propio lugar de trabajo, utilizan un alquiler de servicio y cancelan a un proveedor para que éste sea quien tenga todo ese equipamiento, ya sean discos duros o equipamiento de redes (Abelar \& Hernández, 2010) y, se encargue de toda la mantención y optimización de dicho equipamiento, el ejemplo de empresa que ofrece este tipo de servicio es Amazon Web Service con su servicio EC2 (Amazon Elastic Compute Cloud), es un "servicio web que proporciona tamaño variable de capacidad de cómputo en la nube y, está diseñado para hacer escala informática web más fácil para los desarrolladores” (Abelar \& Hernández, 2010)

- Plataforma como servicio: Se encuentra muy ligada a la capa "software como servicio, porque es la plataforma en donde se envuelve el software que pone a disposición el proveedor y es el medio de virtualización para el hardware que el cliente arrienda. La empresa que más servicios enfocados a esta capa es Google App Engine, el cual permite desarrollar, compartir y alojar aplicaciones Web de terceros en su vasta infraestructura (Abelar \& Hernández, 2010)

- Software como servicio: El proveedor de servicio pone a disposición de los clientes su propio software, creando así un ahorro para el cliente en la búsqueda de licencias, manteniendo actualizado siempre el sistema que necesita el cliente, un ejemplo de esta modalidad es Zetasoftware. En otro campo de la investigación se encontró que existen tres tipos de nubes que se utilizan en el mundo empresarial, entre ellas tenemos:

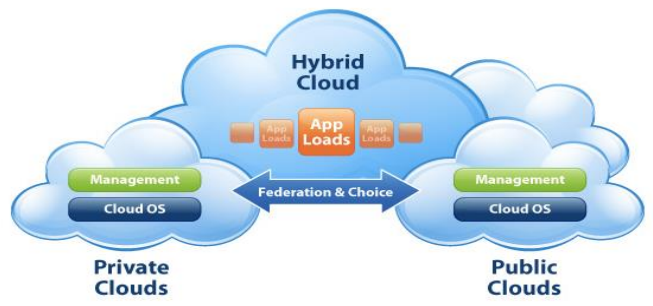

Imagen 1: Tipos de Cloud Computing

Fuente y elaboración: Abelar, M., \& Hernández, I. (2010). Cloud Computing como solución de la gestión de las Pymes. Montevideo: Repositorio Universidad de la República

- Privadas: Las nubes privadas son manejadas por un solo cliente que controla qué aplicaciones debe correr y dónde, son propietarios del servidor, red y disco y que pueden decidir qué usuarios están autorizados a utilizar la infraestructura (Abelar \& Hernández, 2010)

- Públicas: Las nubes públicas se manejan por terceras partes, y los trabajos de muchos clientes de diferentes pueden ser mezclados en los servidores, los sistemas de almacenamiento y otras infraestructuras de la nube. Es importante indicar que los usuarios finales no conocen qué trabajos de otros clientes pueden estar corriendo en el mismo servidor, red, discos como los suyos.

- Híbridas: Las nubes híbridas son las más recomendadas para las empresas pequeñas, combinan los modelos de privada y pública, el propietario es dueño de una parte y comparte otras, actualmente las empresas perciben atracción por la nube híbrida por la facilidad de acoplamiento que tiene a las necesidades de los clientes.

\section{Ventajas del Cloud Computing}


Cloud Computing tiene muchas ventajas, entre las principales para el análisis de nuestro estudio se encontró:

- Acceso: el usuario puede acceder a la información desde otra parte del mundo a cualquier hora.

- Costos Bajos: no existe la compra de licencia para el usuario, no existe compra y gasto en equipamiento de hardware y software, así también el usuario no tiene que preocuparse del mantenimiento ni actualización de sus equipos.

- Espacio de Almacenamiento: el cliente no utiliza espacio físico dentro de su empresa, y los archivos al encontrarse en la nube permitirán que el ordenador del cliente siempre se encuentre libre.

\section{Desventajas de la Cloud Computing}

Cloud Computing trae ventajas significativas, pero es muy importante saber que desventajas puede traer al manejarlo de manera incorrecta

- Dependencia: En la utilización del Cloud Computing el usuario depende del proveedor, como lo indicó Steve Wozniak, cofundador de Apple ante esta creciente inquietud exclamó: “Cuanto más transfiramos todo a la web, en la nube, menos control vamos a tener de ello” (Mamani, 2014), es decir que si el proveedor por ejemplo se declararé en bancarrota y detuviese la provisión de servicios, el cliente podría experimentar serios inconvenientes en el acceso a sus datos y en consecuencia afectar la continuidad del negocio.

- Conexión a Internet: Una desventaja importante para el usuario podría ser qué al no contar con una conexión a internet, no podría acceder a la nube, ni a sus datos (Mamani, 2014); por ello como recomendación el cliente debe contar con un acceso a internet fiable y que la conexión no sea lenta.

- Riesgo: La seguridad y privacidad para el cliente es una preocupación constante, muchas empresas no están cómodas sabiendo que sus datos están almacenados en un servidor virtual, del cual no se sabría con qué nivel de seguridad se cuenta (Abelar \& Hernández, 2010)

- Migración: Si el usuario quiere cambiar su información a otro proveedor del servicio no será fácil transferir gran cantidad de datos de un proveedor a otro.

Principios de la seguridad de la información - riesgos y amenazas

A través del estudio bibliográfico se identificó el informe presentado por INTECO CERT quien hace referencia a informes emitidos CSA (Cloud Security Alliance) (Mejía, 2015):

"las preocupaciones que derivan de estos informes se centran es aspectos como la gestión de los datos, fundamentalmente en la propiedad de los mismos y la forma de operarlos y tratarlos por parte de los proveedores, así como en la identificación y control de acceso a los recursos"

A través del estudio bibliográfico del presente autor, a continuación, se muestra una tabla de riesgos y amenazas más comunes que se presentan a la hora de implementar "computación en la nube": 
- Abuso y mal uso del Cloud Computing

$\checkmark$ Los servicios más afectados son el IaaS, PaaS

$\checkmark$ Registro de acceso a las infraestructuras / plataformas poco restrictivo

$\checkmark$ Proliferación de spammers, creadores de código malicioso

- Amenazas Internas

$\checkmark$ Provocado por empleados descontentos o accidentes por error o desconocimiento.

$\checkmark$ Brechas de seguridad en el manejo de accesos de altas y bajas de acceso, no existe coordinación entre el proveedor y el cliente para mantener los actualizados.

- Pérdida o fuga de información

$\checkmark$ Los datos e información son comprometidos, no existen respaldos ante un evento, no existe cifrado, generación de claves, mecanismos de eliminación y almacenaje de información, políticas de copias de seguridad, acuerdo de confidencialidad.

- Secuestro de sesión o servicio

$\checkmark$ Obtención de credenciales por parte de un atacante

$\checkmark$ Acceso no autorizado a actividades, transacciones, manipulación de datos, pérdida de integridad.

$\checkmark$ Suplantación de información, sitios o portales

\section{- Localización de los datos}

$\checkmark$ Desconocimiento de la ubicación de datos e información

$\checkmark$ Falta de firmas de acuerdo con el proveedor para que los datos se sometan a legislaciones nacionales

$\checkmark$ Desconocimiento de normativas internacionales sobre información y datos que se encuentran en otros países.

\section{La Seguridad de la Información en las empresas}

La Asociación española para la calidad define a la seguridad de la información de la siguiente manera:

"La Seguridad de la información tiene como fin la protección de la información y de los sistemas de la información del acceso, uso, divulgación, interrupción o destrucción no autorizada" (Mejía, 2015)

El término de seguridad de información surge por la necesidad de proteger la información y a los sistemas que la administran, los cuales pueden ser bienes físicos o informáticos. La seguridad de la información también "es un conjunto de reglas, planes y acciones que permiten asegurar la información manteniendo las propiedades de confidencialidad, integridad y disponibilidad" (Valarezo, 2013)

Normas, estándares, metodologías orientadas a la seguridad de la información

Es importante indicar que al obtener las compañías un certificado ISO 27001, ofrecen evidencias (emitidas por un ente certificador) que protegen la información de acuerdo con la 
principal norma en seguridad de la información. Para el presente artículo se ha considerado algunas normas para la Seguridad de la Información, entre ellas tenemos:

\section{- ISO 22301}

British Standard Institution, hace referencia a la nueva norma ISO 22301 como base para la Gestión de la continuidad del Negocio, estableciendo principios, procesos y terminología (Tascán, 2013)

\section{- ISO/IEC 27000}

"A semejanza de otras normas ISO, ISO/IEC 27000 es un conjunto de estándares desarrollados o en fase de desarrollo, por ISO (International Organization for Standardization) e IEC (International Electrotechnical Commission), que proporcionan un marco de gestión de la seguridad de la información utilizable por cualquier tipo de organización, pública o privada, grande o pequeña" (ISO 2700, 2012)

- ISO 27000 cuenta con una serie de recomendaciones divididas en varios documentos, tales como:

$\checkmark$ ISO 27000: Visión General de SGSI

$\checkmark$ ISO 27001: Requerimientos del SGSI

$\checkmark$ ISO 27002: Directrices y Principios Generales (Code of Practice for Information Security Management)

$\checkmark$ ISO 27005: Directrices para la Gestión de Riesgos de Seguridad de la Información

$\checkmark$ ISO 27035: Mejores prácticas para la Gestión de Incidentes de Seguridad de la Información.

\section{- COBIT}

Para la consultora Mexicana ITERA Cobit se define como:

"COBIT es una metodología aceptada mundialmente para el adecuado control de proyectos de tecnología, los flujos de información y los riesgos que éstas implican" (Valarezo, 2013)

La metodología COBIT se utiliza para planear, implementar, controlar y evaluar el gobierno sobre TIC, incorporando objetivos de control, directivas de auditoría, medidas de rendimiento y resultados, factores críticos de éxito y modelos de madurez. Es importante indicar que para el estudio de los posibles riesgos que puedan existir se debe trabajar con una matriz para poder socializarla de la mejor manera, a través del estudio bibliográfico se presenta una guía para identificar los datos menos críticos, entre los cuales tenemos:

- Definir los procesos y flujos de información

- Definir los archivos de información relacionados con los procesos críticos del negocio.

- Asociar los activos de la información en términos de confidencialidad, integridad y disponibilidad.

- Identificar amenazas y vulnerabilidades

- Realizar un análisis de riesgos en base a la probabilidad de ocurrencia e impacto económico (asociado a amenazas y vulnerabilidades) 
- Identificar la clasificación de la información en base a criticidad y sensibilidad de la información resultante, considerando los siguientes niveles:

Según su clasificación:

$\checkmark$ Confidencial

$\checkmark$ Restringida

$\checkmark$ Pública

Según su sensibilidad:

$\checkmark$ Vital

$\checkmark$ No vital

Siguiendo el presente trabajo de investigación a continuación, se presentan las siguientes

\section{Conclusiones}

En el Ecuador existe un gran número de empresas PYMES, cuyo aporte en el desarrollo de la competitividad del país es bastante significativas, pues las mismas se han convertido en generadores de empleo y apoyando además en el movimiento de la economía. En la actualidad la inversión en TIC ha dejado de ser un gasto innecesario para las empresas, porque hoy se considera uno de los principales factores en la toma de decisiones y generación de ventajas competitivas.

Las medidas de seguridad de la información, se vuelven relevantes al momento de decidirse por empezar una migración hacia la nube y la obtención de datos procedentes del Big Data, en la que los datos pueden encontrarse fuera del perímetro de la empresa, del país en diferentes lugares geográficos por ello es importante que las empresas definan con los proveedores acuerdos de niveles de servicio y protección sobre la información, verifiquen leyes y normativas sobre todo de propiedad y confidencialidad de la información. Actualmente el país cuenta con nuevas políticas para la protección y seguridad de la información a pesar de ello no existen normas definidas específicamente hacia los servicios de Big Data y Computación en la Nube.

El Big Data es una tecnología que no sólo está en desarrollo, la misma es utilizada en la actualidad por empresas como eBay, Amazon, Facebook, Twitter, Netflix, IBM, The New York Times, LinkedIn, AOL, entre las más conocidas. Los campos de aplicación de Big Data son incalculables, por ahora se encuentran limitados exclusivamente a la generación de nuevas ideas de las personas encargadas de desarrollar los proyectos o pensar en ideas de implementación de Big Data en un esquema particular (Lara, 2013), entre los proyectos hechos que más utilizan Big Data se encontró:

- Spotify: Es un servicio comercial de streaming de música que se inició en 2008 y desde entonces se ha registrado más de 24 millones de usuarios activos de los cuales 6 millones son usuarios de pago. Tiene millones de fans en Facebook. Cuenta con más de 20 millones de canciones en línea y cada día 20000 nuevas canciones se agregan a la base de datos. Los 
usuarios crearon más de 1 billón de listas de reproducción y más de \$500 millones de USD han sido pagados a los titulares de derechos desde el lanzamiento de Spotify.

Los usuarios de Spotify crean 600 Gigabytes de datos al día y 150 Gigabytes de datos por día a través de los diferentes servicios. Esto le permite la creación de información valiosa que obtiene de sus usuarios, un ejemplo de ello "fue en el 2013 donde Spotify utilizó los datos streaming para predecir los ganadores de los Premios Grammy de ese año, resultando 4 predicciones correctas de las 6 que realizó" (Lara, 2013)

- Coca Cola: La empresa multinacional utiliza el Big Data para producir jugo de naranja con sabor consistente todo el año, aunque las naranjas utilizadas tienen una temporada alta de crecimiento de sólo tres meses. Para ello han desarrollado un algoritmo llamado el "Black Book Model", que

“combina varios conjuntos de datos, tales como imágenes satelitales, fecha de clima, las cosechas esperadas, las presiones de costos, preferencias de los consumidores regionales, información detallada acerca de la gran variedad de los 600 sabores diferentes que conforman una naranja” (Lara, 2013).

La arquitectura del algoritmo es una de las más complejas aplicaciones de análisis de negocios, porque combina hasta 1 trillón de variables de decisión para entregar consistentemente la mezcla óptima.

\section{Recomendaciones}

Se debe mantener una formalización previa de los procesos institucionales, pues de ellos depende el éxito de un proyecto de tecnología, considerar adecuadamente los costos relacionados a tecnología, personas. Implementación con la finalidad de determinar costos ocultos.

El estudio realizado propone los lineamientos básicos y claves para iniciar la implementación de Cloud Computing y Big Data en las empresas Pymes, por lo cual se recomienda en base al análisis desarrollado iniciar procesos y automatización de información menos crítica, para que de manera progresiva y según los controles y mecanismos de seguridad que ofrece el proveedor, ir incrementando hacia los puntos más críticos.

En base de los lineamientos realizar un análisis de los acuerdos de niveles de servicio y confidencialidad que deben estar acorde al negocio y al tiempo que pueden estar si operar por falta de acceso a la información o a los sistemas relacionados, adicionalmente procesos manuales en caso de no contar con la disponibilidad del servicio, exigir al proveedor seguridad de la información y realizar auditorías periódicas del servicio.

\section{Bibliografía}

Abelar, M., \& Hernández, I. (2010). Cloud Computing como solución de la gestión de las Pymes. Montevideo: Repositorio Universidad de la República .

Aguirre, G., \& Andrade, H. (2006). BI Business Intelligence. Guayaquil: Espol. 
Bonales, J., \& Zamora, A. (2015). Variables e índices de Competitividad de las empresas exportadoras, utilizando el PLS. CIMEXUS Vol. X, No. 2, 20.

Calero, A. (2013). Nueva Ciencia y tecnología de Redes . Una nueva forma de tratar la complejidad . TELOS 95, 4.

Crespo, P. (2013). El uso de las TICS como herramienta para la internacionalización de las PYMES en el Ecuador. Cuenca: Repositorio Universidad del Azuay.

Cueva, C. (2014). Deteminación de la línea de base para la aplicación de sistemas BI en el Ecuador. Quito: Repositorio universidad de las fuerzas armadas .

El Comercio. (19 de Julio de 2015). El 82\% de pymes de Ecuador accede a Internet, pero su uso se limita a enviar correos y tareas administrativas, pág. 1.

El Universo. (1 de Junio de 2016). Ecuador es el segundo país que más emprende en el mundo, pero los negocios no se consolidan, pág. 1.

Fernandez, C., \& Piattini, M. (2012). Modelo para el gobierno de las TIC basado en las normas ISO. Madrid: AENORediciones.

ISO 2700. (2012). ISO 27000 . Ginebra: www.iso27000.es/iso27000.html.

Lara, J. (2013). Big Data\&Web Intelligence. Asunción: Repositorio Universidad Católica Nuestra Señora de la Asunción.

Mamani, J. (2014). Ventajas y Desventajas de Cloud Computing. Revista de información, tecnología y sociedad , 3 .

Martinez, S., \& Lara, P. (2014). El Big Data transforma la interpretación de los medios sociales. El profesional de la información v.23, $n 6,6$.

Mejía, J. (2015). Estudio de factibilidad para la implementación eficiente y segura de cloud computing en las empresas pymes del Ecuador. Quito: Repositorio Universidad de las Américas .

Quiroga, L. (2002). Gestión de información, gestión del conocimiento y gestión de la calidad en las organizaciones. ACIMED , 10.

Tascán, M. (2013). Big Data: Pasado, presente y futuro. Telos 95, 4.

Valarezo, F. (2013). Seguridad de la Información y el aseguramiento de la calidad. Madrid : Asociación Española para la calidad . 